\title{
(6) OPEN ACCESS \\ High power Doppler ultrasound score is associated with the risk of triangular fibrocartilage complex (TFCC) tears in severe rheumatoid arthritis
}

\author{
Jen-Hung Chen, ${ }^{1}$ Kuo-Chin Huang, ${ }^{1}$ Chung-Cheng Huang, ${ }^{2}$ Han-Ming Lai, ${ }^{3}$ \\ Wen-Yi Chou, ${ }^{1}$ Ying-Chou Chen ${ }^{3}$
}

${ }^{1}$ Department of Orthopaedic Surgery, Kaohsiung Chang Gung Memorial Hospital, Chang Gung University College of Medicine, Kaohsiung, Taiwan

${ }^{2}$ Department of Radiology, Kaohsiung Chang Gung Memorial Hospital, Chang Gung University College of Medicine, Kaohsiung, Taiwan ${ }^{3}$ Department of Rheumatology, Kaohsiung Chang Gung Memorial Hospital, Chang Gung University College of Medicine, Kaohsiung, Taiwan

\section{Correspondence to \\ Dr Ying-Chou Chen \\ $\mathrm{Sr}_{1}$, Department of \\ Rheumatology Kaohsiung Chang Gung Memorial Hospital and Chang Gung University College of \\ Medicine, Kaohsiung 833, Taiwan; \\ r820713@ms13.hinet.net}

This paper was presented at the World Federation for Ultrasound in Medicine and Biology Congress 2017 in Taipei, China, and published in abstract form in Ultrasound in Medicine and Biology as the Congress book.

Accepted 9 August 2018 Published Online First 29 August 2018

Check for updates

(C) American Federation for Medical Research 2019. Re-use permitted under CC BY-NC. No commercial re-use. Published by BMJ.

To cite: Chen J-H, Huang $\mathrm{K}-\mathrm{C}$, Huang $\mathrm{C}-\mathrm{C}$, et al. J Investig Med 2019;67:327-330.

\section{ABSTRACT}

In the distal radioulnar joint, the triangular fibrocartilage complex (TFCC) is an important stabilizer and are frequently found in patients with rheumatoid arthritis (RA) with wrist pain. This study was designed to predict TFCC tears using high-resolution ultrasound in severe RA. We retrospectively reviewed patients with severe RA. $M R I$ and ultrasound were performed at baseline and after 1 year of follow-up. TFCC tears were recorded. The predictive factors for TFCC tears were analyzed by logistic regression. During the 1-year follow-up period, 54 patients were enrolled (42 females and 12 males), of whom 21 (38.9\%) developed TFCC tears. The body mass index was $22.81 \pm 2.59 \mathrm{~kg} /$ $\mathrm{m}^{2}$ in the TFCC tear group compared with $23.61 \pm 2.76 \mathrm{~kg} / \mathrm{m}^{2}$ in the non-tear group $(p=0.136)$. The mean age was $55.14 \pm 9.54$ years in the TFCC tear group compared with $56.45 \pm 14.04$ years in the non-tear group ( $p=0.596)$. The tear group had a higher Disease Activity Score in 28 joints (DAS28) $(6.36 \pm 0.47$ vs $5.58 \pm 0.65, p=0.011)$ and higher power Doppler (PD) ultrasound score at the dorsal radiocarpal joint $(1.90 \pm 1.30$ vs $1.33 \pm 0.99$, $\mathrm{p}=0.011)$ than the non-tear group. We found that high DAS28 (OR 2.96, 95\% Cl 1.95 to 4.50; $\mathrm{p}=0.001$ ) and higher baseline PD score (OR 1.51, $95 \% \mathrm{Cl} 1.07$ to $2.14 ; p=0.019$ ) were significantly associated with a higher risk of TFCC tears by logistic regression. So we conclude a higher wrist PD score in severe RA predicted future TFCC tears. Therefore, we suggest to use PD score in such patients to monitor the risk of future TFCC tears.

\section{BACKGROUND}

In the distal radioulnar joint, the triangular fibrocartilage complex (TFCC) is an important stabilizer.

A TFCC tear is a major cause of wrist pain at ulnar side, especially in patients with rheumatoid arthritis (RA). Palmer classified TFCC tears into four subtypes, ${ }^{1}$ and recent studies have reported the new anatomical structure of TFCC. $^{2} 3$ Tears can be detected using various radiological methods, including arthrography,

\section{Significance of this study}

What is already known about this subject?

- The triangular fibrocartilage complex (TFCC) is an important stabilizer of the distal radioulnar joint.

- Tears can be detected using various radiological methods, including arthrography, MRI, and MR arthrography. TFCCs lesions are associated with diminished function including grip weakness and ulnar-sided wrist pain in rheumatoid arthritis (RA).

What are the new findings?

- Those with TFCC tear had a higher power Doppler (PD) ultrasound score and higher Disease Activity Score in 28 joints (DAS28) than the non-tear group.

- Logistic regression analysis showed that a higher baseline PD score and high DAS28 were significantly associated with a higher risk of TFCC tears.

How might these results change the focus of research or clinical practice?

- Higher PD score at wrist joint predicted future TFCC tears in patients with severe RA.

- We suggest to use PD score in patients with severe RA to monitor the risk of future TFCC tears.

MR arthrography and MRI. ${ }^{4}$ TFCC lesions are associated with diminished function including ulnar-sided wrist pain in RA and grip weakness.

This study aimed to evaluate the predictive role of ultrasound for TFCC tears using high-resolution morphological MRI.

\section{METHODS}

\section{Research methodology}

A retrospective case-control study.

\section{Study patients}

We collected patients with RA with high disease activity who had undergone MRI of the wrist. 


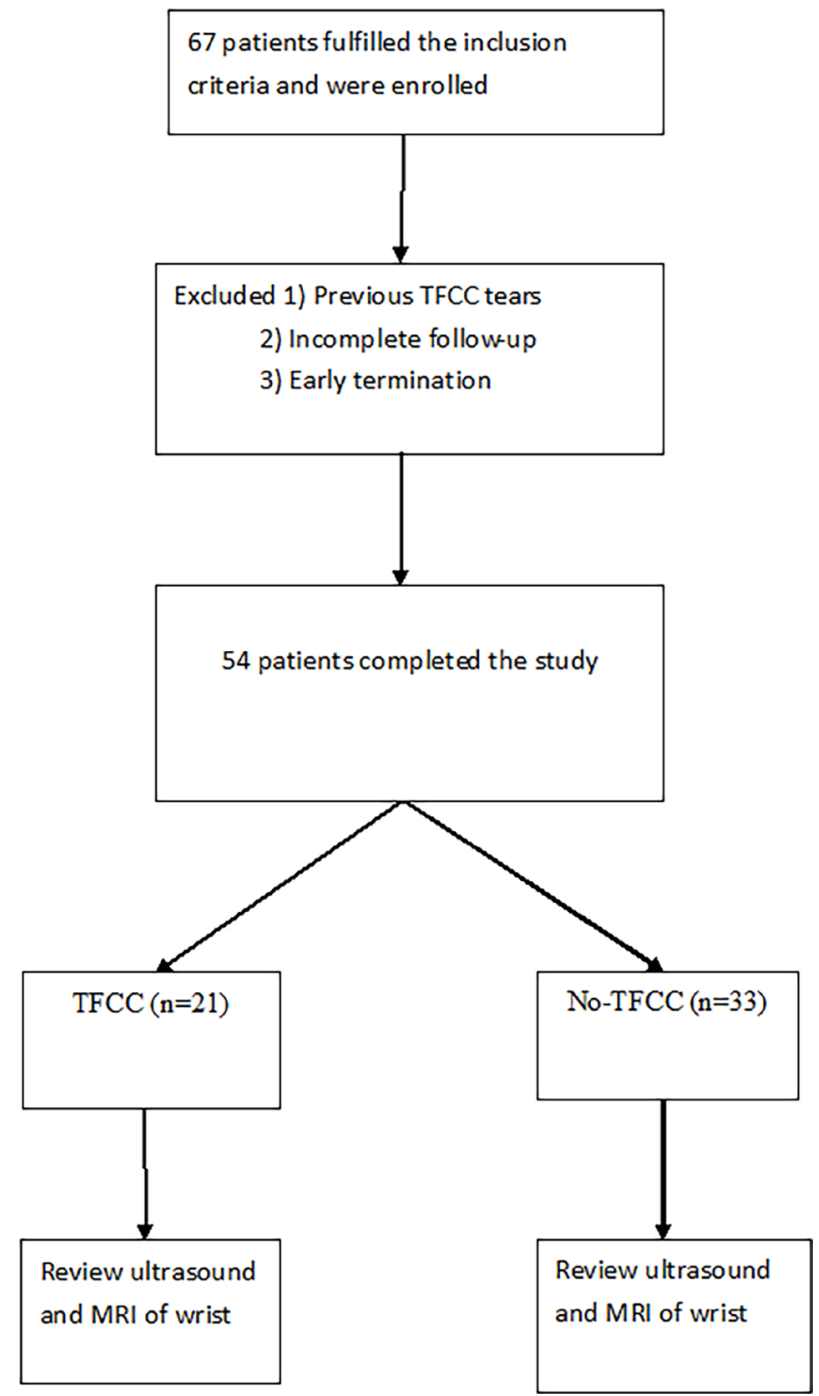

Figure 1 Flow chart of the study. TFCC, triangular fibrocartilage complex.

Cases were patients diagnosed with high RA disease activity with TFCC tears.

Controls were those with high RA disease activity without TFCC tears.

\section{Diagnostic criteria for RA}

The American College of Rheumatology 2010 criteria $^{5}$ for RA were used to enroll the study patients.

Application of these criteria provided a score of $0-10$, with a score of 6 or greater being indicative of the presence of definite RA.

Definition of high RA disease activity

A Disease Activity Score in 28 joints (DAS28) greater than 5.1 was considered to be indicative of high disease activity ${ }^{6}$

\section{Study protocol}

Inclusion criteria

RA with high disease activity and having undergone wrist MRI and ultrasound examinations.

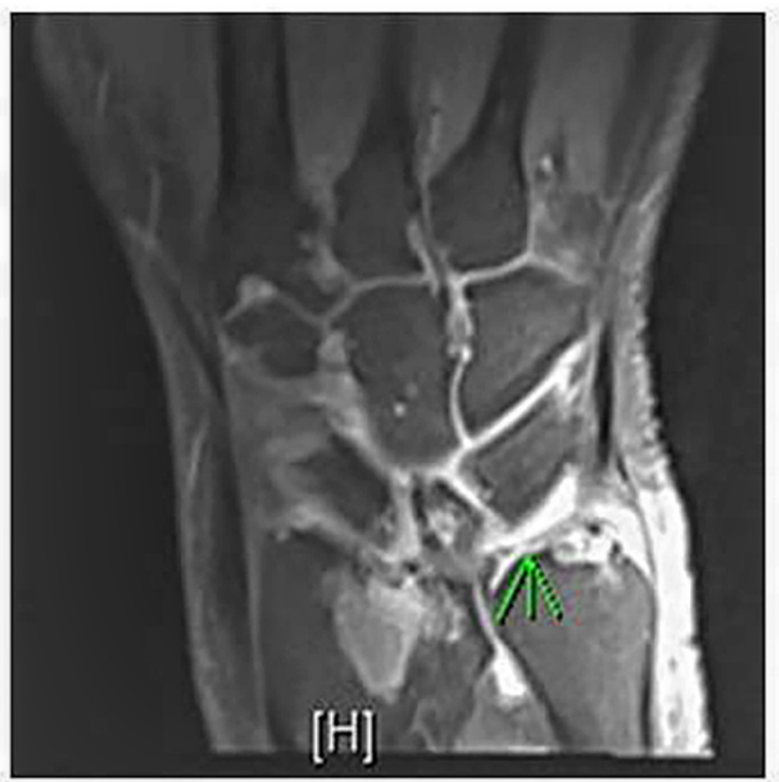

Figure 2 A 60-year-old woman with severe RA. Follow-up MRI showed perforation at the left disc proper of the triangular fibrocartilage complex.

\section{Exclusion criteria}

Patients were excluded from the study if they had the following conditions:

1. Infections.

2. Underlying neoplasms or known hematological disorders that affected thrombosis, platelet count, or function.

3. Major systemic diseases such as end-stage renal disease, liver cirrhosis, or congestive heart failure.

\section{Ultrasound assessment}

Bilateral wrist radiocarpal joints were assessed for the presence of synovial power Doppler (PD) signals.

The synovial PD signals were scored on a semiquantitative scale of $0-3$ ( 0 : absent (no synovial flow); 1 : mild ( $\leq 3$ PD signals); 2: moderate ( $>3$ PD signals in less than half of the synovial area); 3 : marked (signals in more than half of the synovial area)).

\section{MRI}

Complete MRI findings at baseline and after 1 year of follow-up were reviewed. The TFCC was classified as either being normal or torn (a full-thickness tear). A tear was diagnosed on imaging if there was evidence of associated leakage of intra-articular contrast material from the radiocarpal joint into the inferior radioulnar joint or disruption of fibers.

Abnormal MRI findings included: fluid intensity extending to the tissue surface on either or both sides, or tissue transection or irregular high intensity with morphological alterations. Those with baseline TFCC tears were excluded from the study.

\section{RESULTS}

Sixty-seven patients underwent MRI and ultrasound examinations of the wrist. Of these patients, 13 had previous 


\begin{tabular}{|c|c|c|c|}
\hline Variables & TFCC $(n=21)$ & $\begin{array}{l}\text { No-TFCC } \\
(n=33)\end{array}$ & $P$ values \\
\hline Age (years) & $55.14 \pm 9.66$ & $56.45 \pm 14.15$ & 0.711 \\
\hline Body mass index $\left(\mathrm{kg} / \mathrm{m}^{2}\right)$ & $22.81 \pm 2.62$ & $23.61 \pm 2.78$ & 0.296 \\
\hline Gender (female, \%) & $21(100 \%)$ & $21(63.6 \%)$ & 0.001 \\
\hline Anti-CCP (No, \%) & $21(100 \%)$ & $27(81.8 \%)$ & 0.003 \\
\hline $\mathrm{RF}(\mathrm{No}, \%)$ & $18(85.7 \%)$ & $24(72.7 \%)$ & 0.155 \\
\hline ANA (No, \%) & $12(57.1 \%)$ & $15(45.5 \%)$ & 0.324 \\
\hline DAS28 & $6.36 \pm 0.47$ & $5.58 \pm 0.65$ & 0.001 \\
\hline Baseline PD score & $3.8 \pm 1.3$ & $2.6 \pm 0.99$ & 0.011 \\
\hline
\end{tabular}

ANA, antinuclear antibody; CCP, cyclic citrullinated peptide; DAS28, Disease Activity Score in 28 joints; PD, power Doppler; RF, rheumatoid factor; TFCC, triangular fibrocartilage complex.

TFCC tears or terminated the study early and 54 completed the study (figure 1).

There were 42 females and 12 males. During the follow-up period, 21 (38.9\%) developed TFCC tears (figure 2). The mean age in the TFCC tear and non-tear group was $55.14 \pm 9.54$ and $56.45 \pm 14.04$, respectively $(p=0.596)$. The body mass index in the TFCC tear and non-tear group was $22.81 \pm 2.59 \mathrm{~kg} / \mathrm{m}^{2}$ and $23.61 \pm 2.76 \mathrm{~kg} / \mathrm{m}^{2}$, respectively $(\mathrm{p}=0.136)$. The tear group had a higher DAS28 $(6.36 \pm 0.47$ vs $5.58 \pm 0.65, \mathrm{p}=0.011)$ and higher PD ultrasound score at the dorsal radiocarpal joint $(1.90 \pm 1.30$ vs $1.33 \pm 0.99$, $\mathrm{p}=0.011)$ than the non-tear group (table 1). We found a higher baseline PD score (OR 1.51, 95\% CI 1.07 to 2.14; $\mathrm{p}=0.019)$ and high DAS28 $(\mathrm{OR}=2.96,95 \% \mathrm{CI} 1.95$ to $4.50 ; \mathrm{p}=0.001$ ) were significantly associated with a higher risk of TFCC tears (table 2).

\section{DISCUSSION}

In this study, 21 of the 54 (38.9\%) patients developed TFCC tears, which is a very high proportion of the patients with severe RA. However, this may be due to selection bias. In this retrospective review, only those with severe wrist pain underwent MRI examinations. Radiological evaluations of the TFCC included X-ray, CT, or MRI. The TFCC has been reported to have a similar picture to the knee meniscus in MRI, ${ }^{7}$ and MRI has been proposed to be a non-invasive alternative diagnostic test. ${ }^{8}$

MRI can provide additional information regarding bone edema, cartilage lesions, neuropathy, and bone and muscle injuries. All of the TFCC tears were proven by MRI in this study, which is a reliable method to confirm TFCC. In this study, higher RA activity including higher DAS28 and higher

Table 2 Multivariate analysis of the factors to predict TFCC

\begin{tabular}{lllll}
\hline & $\begin{array}{l}\text { Regression } \\
\text { coefficient }\end{array}$ & SE & P values & OR $(95 \% \mathrm{Cl})$ \\
\hline Gender & -0.241 & 0.411 & 0.557 & $0.79(0.35$ to 1.76$)$ \\
$\begin{array}{l}\text { Body mass } \\
\text { index }\left(\mathrm{kg} / \mathrm{m}^{2}\right)\end{array}$ & -0.091 & 0.048 & 0.060 & $0.91(0.83$ to 1$)$ \\
$\begin{array}{l}\text { Baseline PD score } \\
\text { Anti-CCP }\end{array}$ & 0.414 & 0.176 & 0.019 & $1.51(1.07$ to 2.14$)$ \\
DAS28 & 0.303 & 0.328 & 0.356 & $1.35(0.71$ to 2.57$)$ \\
\hline
\end{tabular}

CCP, cyclic citrullinated peptide; DAS28, Disease Activity Score in 28 joints; $P D$, power Doppler; TFCC, triangular fibrocartilage complex.
PD scores were associated with a risk for TFCC tears. In RA, structural joint damage may be caused by synovitis ${ }^{9}$; therefore, we must focus on synovitis to monitor disease activity. It has been reported that synovitis includes periarticular vasodilatation and synovial proliferation, followed by angiogenesis and invasive behavior of rheumatoid pannus. This may explain the higher proportion of TFCC in patients with severe RA.

Using PD score, we found a relationship between joint inflammation activity and synovial vascularization and this could predict TFCC tears. High-resolution ultrasound had been used to monitor RA. PD allows for the assessment of synovial vascularity and to differentiate between inflamed and non-vascular synovial swelling.

Taylor $e t a l^{10}$ evaluated the prognostic value of ultrasound in patients with RA receiving antitumour necrosis factor therapy and found that baseline synovial vascularization detected by PD in joints was correlated with radiographic joint damage over the following year. Apart from ultrasound, erythrocyte sedimentation rate (ESR), C-reactive protein (CRP) and DAS28 can be used for clinical follow-up. However, severe RA is associated with high DAS28, ESR and CRP levels; so when treating this group of patients, it can be difficult to predict radiological progression using these markers alone.

There are several limitations to this study. First, the patients were treated with various disease-modifying antirheumatic drugs (DMARDs), oral corticosteroids and non-steroid anti-inflammatory drugs at variable doses during the study because it was conducted in accordance with daily clinical practice. Therefore, we could not compare the predictive role of PD ultrasound variables based on the prescribed DMARDs because the therapeutic decisions were made without the previous knowledge of the sonographic findings, and we cannot evaluate the potential effect of different DMARDs on PD ultrasound parameters or study the effect of PD ultrasound findings when making therapeutic decisions.

\section{CONCLUSION}

This study found that in patients with RA, the TFCC not only had degenerative change but also inflammation was found; so there was a high proportion of TFCC tears. In addition, the results showed that a higher PD score at wrist joint predicted future TFCC tears in the patients with RA. Therefore, we recommended using PD score in patients with severe RA to monitor possible future TFCC tears.

Contributors $\mathrm{J}-\mathrm{HC}$ and $\mathrm{K}-\mathrm{CH}$ designed and performed the research. $\mathrm{C}-\mathrm{CH}$ performed the MRI evaluation. H-ML, W-YC and Y-CC provided rheumatoid arthritis care. Y-CC analyzed the data and wrote the final article.

Funding This study was sponsored by Kaohsiung Chang Gang Memorial Hospital CMRPG8G1351.

Competing interests None declared.

Patient consent Not required.

Ethics approval This clinical investigation was conducted with the approval of our institution Kaohsiung Chang Gang Memorial Hospital review board and it was conducted in accordance with the Declaration of Helsinki and the International Conference on Harmonization of Good Clinical Practice Guidelines. According to Taiwan law, no additional informed consent was required, and patient information was anonymized and de-identified before data analysis. 
Provenance and peer review Not commissioned; externally peer reviewed.

Open access This is an open access article distributed in accordance with the Creative Commons Attribution Non Commercial (CC BY-NC 4.0) license, which permits others to distribute, remix, adapt, build upon this work noncommercially, and license their derivative works on different terms, provided the original work is properly cited, appropriate credit is given, any changes made indicated, and the use is non-commercial. See: http://creativecommons. org/licenses/by-nc/4.0/.

\section{REFERENCES}

1 Gross SC, Watson HK, Strickland JW, et al. Triquetral-lunate arthritis secondary to synostosis. J Hand Surg Am 1989;14:95-102.

2 Nakamura T, Yabe Y, Horiuchi Y. Functional anatomy of the interosseous membrane of the forearm - dynamic changes during rotation. Hand Surg 1999;4:67-73.

3 Nakamura T, Makita A. The proximal ligamentous component of the triangular fibrocartilage complex. J Hand Surg Br 2000;25:479-86.
4 Rüegger C, Schmid MR, Pfirrmann CW, et al. Peripheral tear of the triangular fibrocartilage: depiction with MR arthrography of the distal radioulnar joint. AJR Am J Roentgenol 2007;188:187-92.

5 Villeneuve E, Nam J, Emery P. 2010 ACR-EULAR classification criteria for rheumatoid arthritis. Rev Bras Reumatol 2010;50:481-3.

6 Walker D, Hall S. NICE guidance on rheumatoid arthritis: implications and challenges for rheumatologists. Rheumatology 2010;49:619-20.

7 Oneson SR, Timins ME, Scales LM, et al. MR imaging diagnosis of triangular fibrocartilage pathology with arthroscopic correlation. AJR Am J Roentgenol 1997;168:1513-8

8 Blazar PE, Chan PS, Kneeland JB, et al. The effect of observer experience on magnetic resonance imaging interpretation and localization of triangular fibrocartilage complex lesions. J Hand Surg Am 2001;26:742-8.

9 Conaghan PG, O'Connor P, McGonagle D, et al. Elucidation of the relationship between synovitis and bone damage: a randomized magnetic resonance imaging study of individual joints in patients with early rheumatoid arthritis. Arthritis Rheum 2003;48:64-71.

10 Taylor PC, Steuer A, Gruber J, et al. Comparison of ultrasonographic assessment of synovitis and joint vascularity with radiographic evaluation in a randomized, placebo-controlled study of infliximab therapy in early rheumatoid arthritis. Arthritis Rheum 2004;50:1107-16. 